\section{Hypothalamic Glioma}

\section{Robert Rider}

Department of Psychology, Drexel University, Philadelphia, PA, USA

\section{Definition}

Hypothalamic glioma is a tumor originating in the hypothalamus gland of the brain. These types of tumors can produce a range of symptoms, which include changes in growth rate, hyperactivity, euphoria, loss of appetite, disruptions of vision, and headache. Seizures may occur as a result of a hypothalamic glioma. The course of tumor growth may be slow or rapid, and tumors may remain static in size for years. Visual field testing can aid in tracking the rate of tumor growth.

\section{References and Readings}

Armstrong, C. L., Schmus, C. J., Belasco, J. B. (In press). Neuropsychological problems in neuro-oncology. In C. L. Armstrong (Ed.), Handbook of medical neuropsychology: Applications of cognitive neuroscience. New York: Springer. 